\title{
Modeling nonlinear relationships in ERP data using mixed-effects regression with $\mathrm{R}$ examples
}

\author{
ANTOINE TREMBLAY AND AARON J. NEWMAN \\ NeuroCognitive Imaging Laboratory, Dalhousie University, Halifax, Nova Scotia, Canada
}

\begin{abstract}
In the analysis of psychological and psychophysiological data, the relationship between two variables is often assumed to be a straight line. This may be due to the prevalence of the general linear model in data analysis in these fields, which makes this assumption implicitly. However, there are many problems for which this assumption does not hold. In this paper, we show that, in the analysis of event-related potential (ERP) data, the assumption of linearity comes at a cost and may significantly affect the inferences drawn from the data. We demonstrate why the assumption of linearity should be relaxed and how to model nonlinear relationships between ERP amplitudes and predictor variables within the familiar framework of generalized linear models, using regression splines and mixed-effects modeling.
\end{abstract}

Descriptors: EEG, ERP, Nonlinear relationships, GAMM, Regression splines

Event-related potential (ERP) datasets are commonly collected in cognitive neuroscience experiments because they offer rich spatiotemporal information about brain activity during perception, cognition, and action. The potential power of ERP data comes with the cost that the datasets tend to be large and quite complex. Thus, the richness of the data demands analytical techniques that are appropriate to fully describe both the complexity of the data, and control for factors that might interfere with such analyses. In trying to deal with such complex data, it is very common to assume that the relationship between ERP amplitudes/latencies and an independent variable is linear. For example, in a study that investigates the relationship between age and amplitude of the N1 ERP component, one would assume that a given increase in age is associated with a consistent increase (or decrease) in the amplitude and/or latency of the N1, regardless of whether the increase is from 23 to 24 years old or from 69 to 70 years old.

In reality, there are many problems for which the answer is not a straight line (Baayen, Kuperman, \& Bertram, 2010; Hastie \& Tibshirani, 1990; Kryuchkova, Tucker, Wurm, \& Baayen, 2012; Pinheiro \& Bates, 2000; Ruppert, Wand, \& Carroll, 2003; Tremblay \& Baayen, 2010; Wood, 2006; H. Wu \& Zhang, 2006), and ERPs are no exception. For example, Carbon, Schweinberger, Kaufmann, and Leder (2005) investigated the effect of "Thatcherized" faces (in

AT was supported during data collection by a Social Sciences and Humanities Research Council of Canada (SSHRC) doctoral fellowship while in the Department of Linguistics at the University of Alberta, Edmonton, Canada (2007-2009). AT was supported during analysis and writing by a SSHRC postdoctoral fellowship in the Department of Psychology and Neuroscience at Dalhousie University, Halifax, Canada (2011-2013). AJN was supported by a SSHRC Standard Research Grant.

Address correspondence to: Antoine Tremblay, NeuroCognitive Imaging Laboratory, Dalhousie University, Halifax, NS B3H 4R2, Canada. E-mail: trea26@gmail.com which the eyes and mouth regions are turned upside down) on N170 amplitudes (a negative component occurring between 130 and $200 \mathrm{~ms}$ at occipitotemporal scalp sites). Pictures of normal and Thatcherized faces were presented at either 0,90 , or $180^{\circ}$ rotation; only when presented upright are these perceived as severely distorted. Carbon et al. (2005) found a nonlinear effect of rotation on N170 amplitudes in response to faces: Upright Thatcherized faces elicited a smaller N170 than Thatcherized faces rotated 90 or $180^{\circ}$. The effect on N170 amplitudes of the latter two orientations, however, did not differ. Boutheina, Coutya, Langer, and Roy (2009) also investigated the effects of picture rotation on N170 amplitudes. They used pictures of normal faces that were rotated $0,22.5,45$, $67.5,90,112.5,157.5$, or $180^{\circ}$ and found a relatively complex nonlinear effect on N170 amplitudes and latencies (see Figure 3B in Boutheina et al., 2009). Numerous other examples of nonlinear effects on ERPs can be found in the literature, in areas such as development (Webb, Long, \& Nelson, 2005), rhythm perception (Pablos Martin, Deltenre, Rossion, Hoonhorst, \& Colin, 2007), and auditory processing (Inui et al., 2010).

The studies mentioned above did not assume linearity, which is a step forward. However, in keeping with the traditional analysis of variance (ANOVA) approach to statistical analysis, they tested for nonlinear relationships using the common practice of discretizing the variables of interest. That is, they have reduced inherently continuous measures, such as rotation angle (or stimulus duration, interstimulus interval, magnitude, frequency, etc.) to factor variables with only a few levels. Such a practice is likely attributable to the natural development of cognitive neuroscience from behavioral approaches to studying cognition that traditionally rely heavily on ANOVA models. However, it comes with problems of its own. Indeed, not only does discretization induce bias into the analysis, but it also decreases statistical power and increases the probability of finding spurious associations (Cohen, 1983; McCallum, Zhang, 
(A)

p-value $=0.41$

R-squared $=0.01 ;$ AIC $=648$

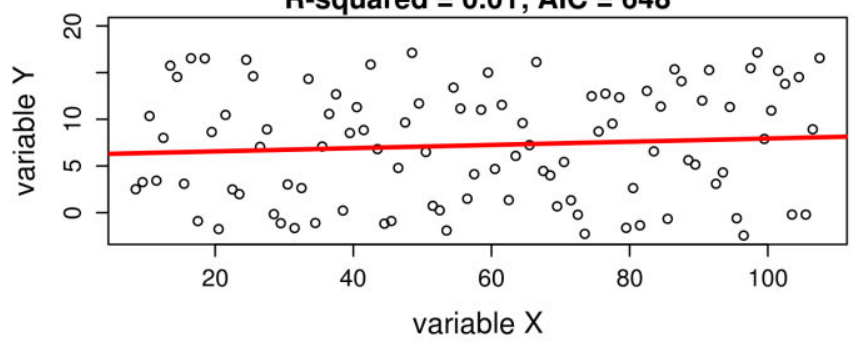

(C)

p-value $<0.001$

R-squared $=0.16 ;$ AIC $=2174$

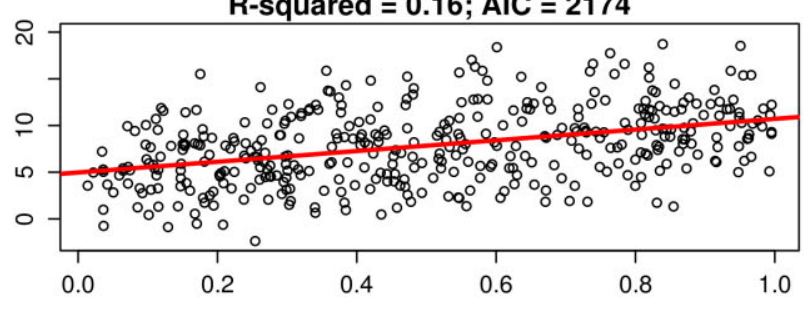

(E)

p-value $=0.27$

R-squared $=0.01 ;$ AIC $=955$

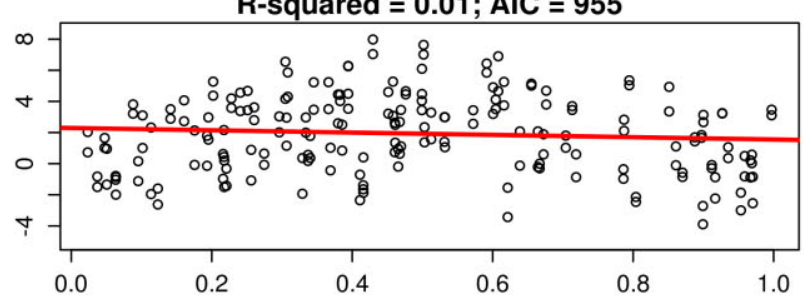

(G)

p-value $<0.001$

R-squared $=0.23 ;$ AIC $=2162$

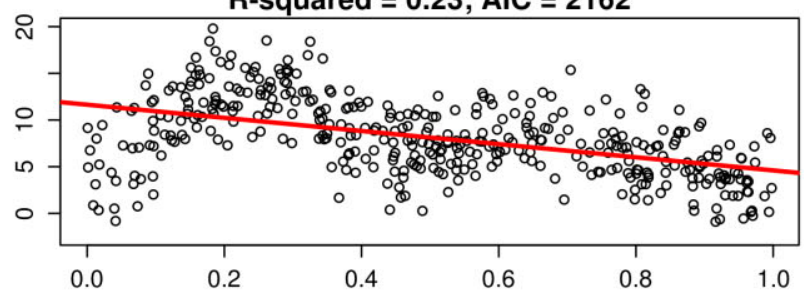

(B)

p-value $=1$

R-squared $=0 ; A I C=646$

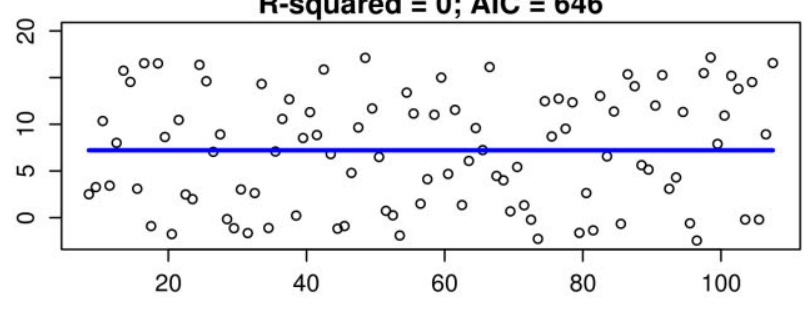

(D)

p-value $<0.001$

R-squared $=0.16 ;$ AIC $=2175$

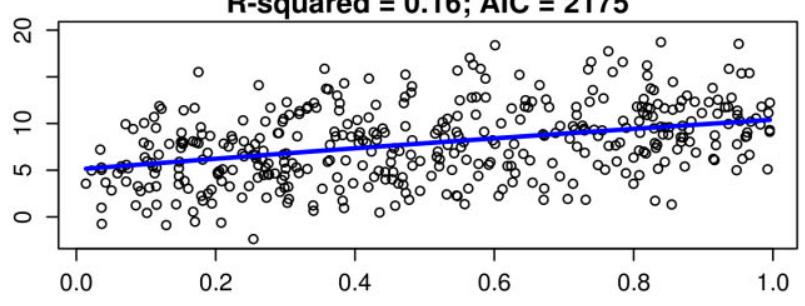

(F)

p-value $<0.001$

R-squared $=0.16 ; A I C=923$

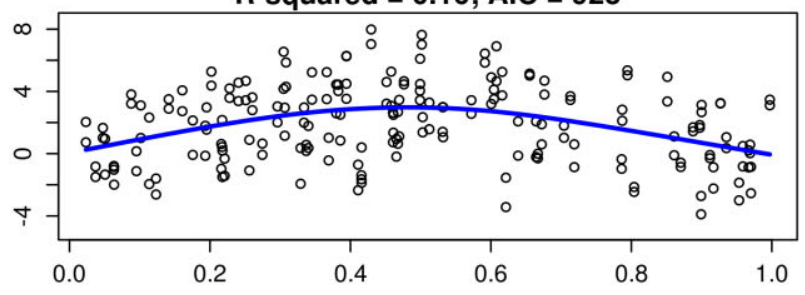

(H)

p-value $<0.001$

R-squared = 0.49; AIC $=2001$

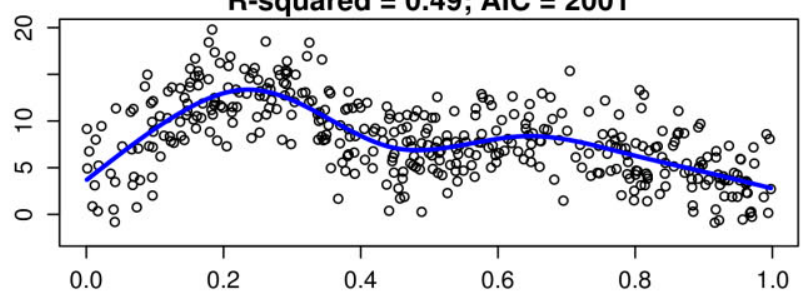

Figure 1. Assuming linearity (left column) versus not assuming linearity (right column).

Preacher, \& Rucker, 2002). Fortunately, such factorization is neither desirable, nor necessary to detect nonlinear relationships. ${ }^{1}$

\section{Relaxing the Assumption of Linearity: A Set of Simple Examples}

Assuming linearity when it does not hold may lead one to (a) overlook important structure in the data, (b) conclude that there is

1. Although we advocate here that continuous variables should not be discretized, there are circumstances in which such variables should be divided into categories. For example, if the values of a variable happen to be distributed in such a way that there are two distinct peaks separated by a large gap, then it would make sense to dichotomize it. It goes without saying that an inherently binomial variable should be entered in a model as a factor variable. no relationship between two variables when in fact there is one, and (c) underestimate the strength of the relationship between a dependent and an independent variable (Sahai \& Ageel, 1997). To illustrate these points, we simulated four data sets (based on the gamSim function from R package mgcv; R Development Core Team, 2013; Wood, 2012) and compared models that assume linearity to ones that do not (for the sake of this example, we will gloss over how the assumption of linearity was relaxed for the latter analyses). In Figure 1, the panels on the left show the models that were fitted assuming linearity, whereas the ones on the right show the models that were fitted without making this assumption. In the first simulated data set, shown in the top panels (A and B), the dependent $(\mathrm{Y})$ variable does not correlate with the predictor variable, and neither of the two models report that it does. The 2-point 
difference in Akaike's Information Criterion (AIC; Akaike, 1973) indicates that both models are equivalent. ${ }^{2}$ Panels $\mathrm{C}$ and D show data in which there is a linear relationship between the response and predictor variables, which is captured by both linear and nonlinear models. Indeed, the nonlinear model fit results in a straight line since there is no nonlinearity in the relationship, and the AIC values indicate that both models are just as likely given the data. The simulated relationship between the dependent and independent variables in panels $\mathrm{E}$ and $\mathrm{F}$ is quadratic. The model for which linearity is assumed (panel E) not only fails to capture the quadratic relationship, but it also falsely concludes that there is no significant relationship between $\mathrm{X}$ and $\mathrm{Y}$. The model shown in panel $\mathrm{F}$, however, is able to capture the quadratic trend, and comparison of the AIC values for the two models confirms that this is a better fit. In panels $\mathrm{G}$ and $\mathrm{H}$, the simulated relationship is more complex, taking on a "wavy" function. Although the model for which linearity is assumed does find that there is a significant relationship between $\mathrm{X}$ and $\mathrm{Y}$, the actual nature of the relationship is only part of the underlying truth. This leads the model to underestimate the strength of the relationship between X and Y, and the accuracy of the prediction varies as a function of $\mathrm{X}$. Conversely, the model for which linearity is not assumed, illustrated in panel $\mathrm{H}$, is able to capture the true nature of the relationship, and correspondingly has a much lower AIC value. Although we advocate here that continuous variables should not be discretized, there are circumstances in which such variables should be divided into categories. For example, if the values of a variable happen to be distributed in such a way that there are two distinct peaks separated by a large gap, then it would make sense to dichotomize it. It goes without saying that an inherently binomial variable should be entered in a model as a factor variable.

\section{Modeling Nonlinear Relationships}

Testing for nonlinear relationships can be seen as a natural extension of testing for linear relationships. Linear regression is familiar to virtually anyone with a modicum of training in statistics, and allows one to test for a linear relationship between two variables as shown in the left-hand columns of Figure 1. Thus, for example, in the analysis of the effects of image rotation in the Carbon et al. (2005) study described above, the levels of rotation could have been treated as values along a continuum of possible rotation angles, rather than as categorical levels. The problem with this approach, as demonstrated in the examples in Figure 1, is that if the relationship is nonlinear then a linear fit will not be optimal. In contrast, pairwise testing of different "levels" of rotation is able to detect which (if any) pairs of levels differ. Certain relationships can also be detected using a set of linear contrast weights, as in Helmert or polynomial contrasts. However, this approach does not generalize easily to many levels of a factor, and because each pairwise or contrast test assumes independence correction for multiple comparisons should be applied-potentially weakening sensitivity to extant effects.

2. AIC is a relative measure that indexes how likely a model is given the data (relative to other models). It is calculated as $2 \times$ the number of parameters in the model $-2 \times$ the $\log$ likelihood of the model (i.e., more complex models will tend to have higher AIC values). Statistical models can be compared by way of AIC, where those with smaller values reflect more likely models. By convention, we consider that two models differ if the difference in AIC value is equal to or greater than 5, meaning that the model with the lower AIC is 12 times more likely given the data than the one with the higher AIC. We refer the interested reader to Burnham, Anderson, and Huyvaert (2011) and Symonds and Moussalli (2011) for more details about AIC and model selection.
Nonlinear relationships can be modeled without discretization by applying a series of linear transformations to the predictor variables. Regression splines, which are thought to be the ideal series of transformations and the most computationally efficient (Wood, 2006, p. 148), are curves made up of sections of polynomials joined together at points termed "knots" so that they are continuous in value, as well as first and second derivatives. The specific spline equation used in the model-fitting algorithm we use here (i.e., generalized additive mixedeffects regression; see below) is from $\mathrm{Gu}$ (2002):

$$
\begin{aligned}
R(x, k)= & \frac{\left[\left(k-\frac{1}{2}\right)^{2}-\frac{1}{12}\right]\left[\left(x-\frac{1}{2}\right)^{2}-\frac{1}{12}\right]}{4} \\
& -\frac{\left[\left(|x-k|-\frac{1}{2}\right)^{4}-\frac{1}{2}\left(|x-k|-\frac{1}{2}\right)^{2}+\frac{7}{240}\right]}{24}
\end{aligned}
$$

where $x$ is a vector of covariate values and $k$ is a knot location.

The spline function merely serves to transform variables. The actual modeling of the data will be performed with generalized additive mixed-effects modeling, which we briefly describe below.

\section{Generalized Additive Mixed-Effects Modeling}

Generalized additive mixed-effects modeling (GAMM) is a relatively recent development in statistics (e.g., Gu \& Wahba, 1991; Hastie \& Tibshirani, 1990; Wood, 2006) and has recently been applied to ERP data in several published papers, including Tremblay (2009), Tremblay and Baayen (2010), and Kryuchkova et al. (2012). ${ }^{3}$ Mixed-effect modeling (e.g., GAMM) is a natural tool for modeling repeated measures (Lin \& Zhang, 1999; H. Wu \& Zhang, 2006; L. Wu, 2010), including repeated measurements in ERP data (Bagiella, Sloan, \& Heitjan, 2000; Vossen, van Breukelen, Hermens, van Os, \& Lousberg, 2011). This type of model captures the dependency between repeated measurementssuch as, for example, within subjects as well as within stimuli-by modeling the variance-covariance matrix of the error term. Details about mixed-effects modeling, as well as its potential advantages over repeated measures ANOVA, can be found in a number of recent papers and books (Baayen, 2008; Baayen, Davidson, \& Bates, 2008; Bagiella et al., 2000; Faraway, 2005; Gelman \& Hill, 2007; Pinheiro \& Bates, 2000; Quené \& van den Bergh, 2008; Vossen et al., 2011; H. Wu \& Zhang, 2006; L. Wu, 2010; Zuur, Ieno, Walker, Saveliev, \& Smith, 2009).

A GAMM is a non-parametric regression model with the general additive structure:

$g\left(\mu_{i j}\right)=\mathbf{X}_{\mathrm{i}}^{*} \theta+f_{1}\left(x_{1 i}\right)+f_{2}\left(x_{2 i}\right)+f_{3}\left(x_{3 i}, x_{4 i}\right)+\ldots+\varepsilon_{i j}+\mathbf{Z}_{\mathrm{ij}} \alpha_{\mathrm{i}}$,

where $\mu_{i j} \equiv \mathbb{E}\left(Y_{i j}\right), Y_{i j}$ is a response variable (e.g., amplitude), $X_{i}^{*}$ is a row in the matrix for a strictly parametric model component, $\theta$ is the corresponding parameter vector, the $f(x)$ 's are smooth functions of the covariates, $\varepsilon_{i j}$ are $N\left(0, \sigma^{2}\right)$ measurement errors, and $\mathbf{Z}_{\mathrm{ij}} \alpha_{\mathrm{i}}$ are $N(0$, $D\left(\sigma^{2}\right)$ ) random effects (Faraway, 2005; Hastie \& Tibshirani, 1990; Keele, 2008; Wood, 2006). The basis we use here for our smooth functions, $f(x)$, are the regression splines defined in Equation 1.

3. Generalized additive (mixed-effects) modeling is a widely accepted data analysis method that has been used in well over a thousand papers from a wide variety of domains of inquiry. See section "Domains of Inquiry in which GAMM has been Used" in supporting information "What's Under the Hood: A Brief Introduction to GAMM." 
Like ANOVA, GAMM also fits within the generalized linear mixed-effects model (GLMM). ANOVA and GAMM differ in that (a) GAMM can model more complex random-effect structure (e.g., crossed, independent random effects; Baayen et al., 2008; Quené \& van den Bergh, 2008); (b) GAMM is robust to violations of sphericity if the correct random-effect structure is used (Baayen et al., 2008; Bagiella et al., 2000; Vossen et al., 2011), eliminating the need to correct for this post hoc using methods that are known to be either overly conservative (Greenhouse-Geisser) or liberal (HuynhFeldt); (c) GAMM enables one to appropriately model imbalanced data (Baayen et al., 2008; Bagiella et al., 2000; Gelman \& Hill, 2007; Vossen et al., 2011), a common situation in ERP data; and (d) the model-fitting objective is augmented by a "wiggliness" penalty enabling one to model nonlinear relationships that do not over- or underfit the data, in a manner that reduces subjectivity and circularity (Kriegeskorte, Simmons, Bellgowan, \& Baker, 2009; Wood, 2006).

To fully make use of these capabilities, data should not be averaged across trials or any other variable. Rather, a GAMM should be fitted on the unaveraged (single trial) data. Beyond allowing robust, accurate estimates of variance, this practice has the added benefit of allowing one to model nonlinear relationships between dependent and independent variables in multidimensional space.

Striking a balance between model fit and model smoothness. By specifying the model in terms of smooth functions rather than detailed parametric relationships, it is possible to avoid otherwise cumbersome and unwieldy models. The wiggliness of a smooth function (i.e., the tradeoff between overand underfitting the data) is controlled by the integrated square of the second derivative of a covariate, $\int_{0}^{1}\left[f^{\prime \prime}(x)\right]^{2} d x$, multiplied by $\lambda$, a smoothing parameter (Wood, 2006). As $\lambda$ tends towards infinity, the estimate of $f(x)$ will become a straight line, whereas $\lambda=0$ will result in an unpenalized (wiggly) estimate. One way of (objectively) determining $\lambda$ is through generalized cross validation (GCV). As Hastie and Tibshirani (1990) put it, "it is a way to let the data show us the appropriate functional form" of a smooth (p. 1; emphasis is theirs). The GCV objective is to minimize the error between the model-predicted value of a missing data point and its real value. In brief, the flexibility of a GAMM is determined by $\lambda$ and GCV rather than by the number and location of spline knots (Wood, 2006).

Number of knots and knot locations. By default, knots are located at a covariate's quantiles. Given that model smoothness is determined by $\lambda$ and GCV, the exact number of knots to use is not generally critical. Nevertheless, it should be large enough that one is reasonably sure of having enough degrees of freedom to represent the underlying "truth" reasonably well, but small enough to maintain reasonable computational efficiency (Wood, 2006, also see the Help page for function choose.k in R).

Probability values for smooth terms. To calculate a smooth's probability value, a Wald statistic $T$ is first computed:

$$
T=f^{\prime} V_{f}^{*} f
$$

where $f$ is the vector of values of a smooth term, and $V_{f}^{*}$ is the rank $r$ pseudoinverse of the corresponding Bayesian covariance matrix $V_{f}$, and $r$ is the estimated degrees of freedom $(e d f)$ for the term (Wood, 2013). $T$ is then compared to a $\chi^{2}$ distribution with degrees of freedom equal to $e d f$ for the term plus $\alpha$ (e.g., 0.05) times the number of estimated smoothing parameters.

Confidence intervals. Smooths have Bayesian confidence intervals around them, which are obtained by taking the quantiles from the posterior distribution of the $f(x)$ 's (Marra \& Wood, 2012).

\section{Goals of the Present Study}

The goal of this paper is to demonstrate how relaxing the assumption of linearity can lead to better modeling of ERP data. We also demonstrate how to model nonlinear relationships using GAMM. This paper has been written in LATEX using Sweave and R, and the source document is provided as online supporting information. The data, a pdf of this paper, the .Rnw file used to write it, and the R code used to generate all of the analyses, tables, and figures presented in this paper are available in package nlEEG. The package is also available from http:// hdl.handle.net/10222/22146. ${ }^{4}$ The paper has a second set of supporting information entitled "What's Under the Hood: ABrief Introduction to GAMM" also available from http://hdl.handle.net/10222/22146. These resources can be used to work through the examples, and potentially act as a starting point for the reader's own forays into GAMM analysis. This paper can, however, be read and understood without viewing the accompanying $\mathrm{R}$ code.

\section{Example ERP Data}

The ERP data used here was collected in the context of an immediate free recall experiment described in Tremblay (2009) and Tremblay and Baayen (2010). The goal of this experiment was to investigate whether frequently used four-word sequences, such as end of the year, I don't really know, and at the same time, may be (de)composed via the application of compositional rules or stored and retrieved as wholes (in which case, they should show effects of the frequency of co-occurrence of the four-word sequence). This was achieved by examining whether the frequency of such phrases affected early ERP components such as the anterior N1 (N1a) and the posterior P1. Both of these components peak approximately 110-150 ms after stimulus presentation, and were previously found to be modulated in studies of single words by linguistic variables including frequency and length (Assadollahi \& Pulvermüller, 2003; Hauk, Davis, Ford, Pulvermüller, \& Marslen-Wilson, 2006; Hauk \& Pulvermüller, 2004; Murphy, Roodenrys, \& Fox, 2006; Penolazzi, Hauk, \& Pulvermüller, 2007; Sereno, Rayner, \& Posner, 1998). Tremblay (2009) reported that higher-frequency four-word sequences indeed elicited more negative $\mathrm{N} 1$ and less positive P1 amplitudes than low-frequency sequences.

However, additional data were collected in this study that were not analyzed in Tremblay (2009) or Tremblay and Baayen (2010). In the present paper, we investigate whether working memory capacity, the length of the second word of a sequence, and the position in a list where a four-word sequence was presented (i.e., whether it was presented first, second, ..., fifth, or sixth in a block), and their interaction affected ERPs in the memory task. ${ }^{5}$

4. The $\mathrm{R}$ code chunks contained in this paper are also available as individual .R files in folder nlEEG/vignettes/Rcode_chunks.

5. The second word of a sequence appeared, more often than not, right where the fixation cross was presented (center of the screen). Thus, the second word of a sequence was the first word that participants saw. It stands to reason that the longer the second word of a sequence, the greater the amplitude of the N1should be, given that longer words elicit larger anterior negativities (Murphy et al., 2006). 
In the analyses presented here, we focused on the N1a as the ERP component of interest. The N1a is known to be sensitive to spatial attention as well as lexical frequency, probability of occurrence, and word length (e.g., Luck, 2005; Murphy et al., 2006; Penolazzi et al., 2007, and references cited therein). For instance, high-frequency words elicit greater N1a amplitudes than lowfrequency words, and longer words elicit larger N1a amplitudes than shorter ones. Because in this paper our focus is on demonstrating nonlinear analysis and comparing it to a linear approach, we limited our analyses to a single electrode (Fz, located along the midline, midway between the nose and the vertex of the head) where the N1a component was maximal, and we do not discuss the implications of our results with respect to the cognitive or linguistic literature. Nevertheless, we do provide the R code to perform a GAMM analysis of the whole scalp, as well as results, in section "GAMM Analysis of Entire Scalp in 80-180 ms Window" in supporting information "What's Under the Hood: A Brief Introduction to GAMM."

\section{Participants}

Ten right-handed female students from the University of Alberta were paid for their participation in the experiment (mean age $=23.4 ; S D=1.6 ; \mathrm{min} / \mathrm{max}=22 / 27$ ). All were healthy native speakers of English, had normal or corrected-to-normal vision, and did not report any neurological deficits. Participants had a mean handedness score (Oldfield, 1971) of 79.5/100 ( $S D=15.8)$. We assessed participants' reading span and working memory capacity (henceforth WMC) using an adaptation of the Daneman and Carpenter (1980) test presented on a PC using E-Prime (Mean WMC score $=73 / 100 ; S D=10.4)$.

\section{Study Design}

The stimulus list consisted of 432 four-word sequences taken from the British National Corpus (Davies, 2004). Some examples are end of the year, I don't really know, at the same time, I have to say, it would be a, at the age of, this is not a, we've got to get, and I think it's the. Frequencies, which were obtained from the Variation in English Words and Phrases search engine (Fletcher, 2008), ranged from 0.03 to 105 occurrences per million. The stimuli were divided into 72 blocks. Each block was divided into 18 trials, where in each trial six four-word sequences were randomly presented one at a time in the middle of the screen with an interstimulus interval of roughly $4,000 \mathrm{~ms}$. Sequences subtended on average $\sim 5^{\circ} \times 0.4^{\circ}$ visual angle; the longest four-word string (becoming increasingly clear that) subtended $\sim 8^{\circ} \times 0.4^{\circ}$ visual angle. At the end of each trial (i.e., after having seen six four-word sequences), participants were prompted to type in as many sequences as they could recall.

\section{Electroencephalogram Recordings and Processing}

Electroencephalogram (EEG) recordings were made with active $\mathrm{Ag} / \mathrm{AgCl}$ electrodes from 32 locations according to the International 10/20 system (www.biosemi.com/headcap.htm) at the midline $(\mathrm{Fz}, \mathrm{Cz}, \mathrm{Pz}, \mathrm{Oz})$ and left and right hemisphere (Fp1, Fp2, AF3, AF4, F3, F4, F7, F8, FC1, FC2, FC5, FC6, C3, C4, T7, T8, CP1, CP2, CP5, CP6, P3, P4, P7, P8, PO3, PO4, O1, O2), as well as the right and left mastoids, and referenced online at the common mode sense active electrode. Electrodes were mounted on a nylon cap. Eye movements were monitored by electrodes placed above and below the left eye and at the outer canthi of both eyes, which were bipolarized offline to yield vertical and horizontal electrooculograms (EOG). Analog signals were sampled at $8192 \mathrm{~Hz}$ using a BioSemi (Amsterdam, The Netherlands) Active II digital 24-bit amplification system with an active input range of \pm $262 \mathrm{mV}$ per bit and were band-pass filtered between 0.01 and $100 \mathrm{~Hz}$. Note that BioSemi uses active electrodes and therefore can tolerate high scalp impedances. ${ }^{6}$ The digitized EEG was initially processed offline using Analyzer version 1.05 (Brain Products $\mathrm{GmbH}$, Gilching, Germany): It was rereferenced to the average of the right and left mastoids, downsampled to $128 \mathrm{~Hz}$ (using an Analyzer script), band-pass filtered from 0.01 to $30 \mathrm{~Hz}$ using a forward-backward filter combination where each of the filters was comprised of a two-pole zero-phase infinite impulse response Butterworth filter, and corrected for eye movements and eye blinks by regressing out the vertical and horizontal EOGs (Gratton, Coles, $\&$ Donchin, 1983). The processed signal was then segmented into epochs of 3,000 ms (1,500 ms before and after stimulus onset). Each epoch was baseline corrected on the 1,500-ms segment immediately preceding stimulus onset. An mp4 movie of the scalp topographies through time can be downloaded from http:// hdl.handle.net/10222/22146.

Figure 2 is an animation of the scalp topography through time. The very first frame of the animation depicts the scalp topography at time $t=141 \mathrm{~ms}$ where the N1a was maximal. It is apparent from the scalp animation, as well as from the bottom panel of Figure 2, that a negative deflection around electrode $\mathrm{Fz}$ - the N1a-began at $t \sim 78 \mathrm{~ms}$, peaked at time $t=141 \mathrm{~ms}(\sim-5 \mu \mathrm{V})$, and returned to baseline at time $t \sim 180 \mathrm{~ms}$. Our window for analysis was thus chosen as $80-180 \mathrm{~ms}$.

\section{Results}

The three predictor variables (fixed effects) under investigation were (1) the position of the four- word sequence in the set of six items presented in a block (position), (2) the length of the second word in the four-word chunk (length), ${ }^{7}$ and (3) the WMC of the participant. These predictor variables were transformed prior to plotting or analysis. We first mean-centered position, length, and WMC by subtracting the mean of a variable from each of its values. The main purpose of this centering was to increase numerical stability and remove any spuriously high correlation that may arise between random intercepts and random slopes as well as between fixed and random variables (Baayen, 2008; Hofman \& Gavin, 1998; Kreft \& De Leeuw, 1998). We first present the results from the behavioral analysis and then move on to the ERP analysis.

\section{Behavioral Results}

We investigated whether length, position, WMC, and their interactions affected the probability of correctly recalling a four-word sequence. In order to be correctly recalled, a four-word sequence had to be recalled exactly. That is, if the target sequence was in the middle of, any response other than in the middle of was considered

6. As mentioned in http://www.biosemi.nl/forum/viewtopic.php?t= 486\&highlight= impedance, for active electrodes, "EEG currents do not (significantly) flow via the electrodes because the input impedance of all current biopotential amplifiers is very high. So, typical electrode impedances (smaller than a few hundred $\mathrm{k} \Omega$ ) do not influence measured EEG voltages." Our impedances were around $25 \Omega$.

7. The second word was chosen because it most often overlapped the center of the monitor where subjects fixated between trials. 



Figure 2. Scalp topography at time $t=141 \mathrm{~ms}$ where the N1a was maximal. Lighter shades of gray indicate negative amplitudes (blue colors in online version) while darker shades of gray indicate positive amplitudes (red colors in online version). In online version, green indicates an amplitude of $0 \mu \mathrm{V}$. The bottom panel shows the average waveform of each of the 32 electrodes overlaid on top of each other (each electrodes is graphed with a different color). The $x$ axis represents time in milliseconds and the $y$ axis is amplitude in $\mu \mathrm{V}$. A vertical black bar marks the peak of the N1a component.

to be incorrect (e.g., in the middle, in the middle and, in the middle of $a$, or at the middle of). However, we accepted minor misspellings such as in the mdle of or $n$ the midle of. The optimal GAMM model is summarized in Table 1. The model included significant Position $\times$ Length and Position $\times$ WMC interactions. Note that, although the summary table states that $p$ value for the Posi-

Table 1. Results of the Behavioral Analysis

\begin{tabular}{llcr}
\hline \hline & $e d f$ & $\chi^{2}$ & $p$ value \\
\hline Pos & 4.6 & 367 & $<.001$ \\
Lngth & 7.8 & 23.3 & .004 \\
WMC & 1 & 4 & .045 \\
Pos:Lngth & 5.5 & 11.3 & .136 \\
Pos:WMC & 8.4 & 30.8 & .001 \\
Lngth:WMC & 1 & 2.5 & .112 \\
Pos:Lngth:WMC & 9.5 & 14.3 & .325 \\
\hline \hline
\end{tabular}

Note . Pos = position; Lngth = length; $\mathrm{WMC}=$ working memory capacity; $e d f=$ estimated degrees of freedom. tion $\times$ Length interaction was greater than .05 , it can be concluded from both the AIC comparison and the $95 \%$ confidence interval (CI) that this interaction should remain in the model. ${ }^{8}$

Results of the behavioral analysis are depicted in Figure 3. In panel A, it can be seen that length had a small effect on the probability of correctly recalling a four-word sequence. More specifically, recall probabilities were greatest when the second word was two to six letters long at positions 5 and 6. Panel B illustrates the Position $\times$ WMC interaction, which exhibited an overall recency effect whereby more recently presented four-word sequences were more likely to be correctly recalled. The recency effect possibly reflected an advantage in activation strength for the sequences that were presented more recently within a block (Jones $\&$ Oberauer, 2013). However, it was more pronounced for participants with higher working memory capacity scores. Furthermore, people with a WMC score below 0.7 did not reliably recall

8. We do not consider the three-way Position $\times$ Length $\times$ WMC or the two-way Length $\times$ WMC interactions given that $p>.05$ and they were not warranted by way of AIC comparisons. 


\section{(A) Position X LengthB}

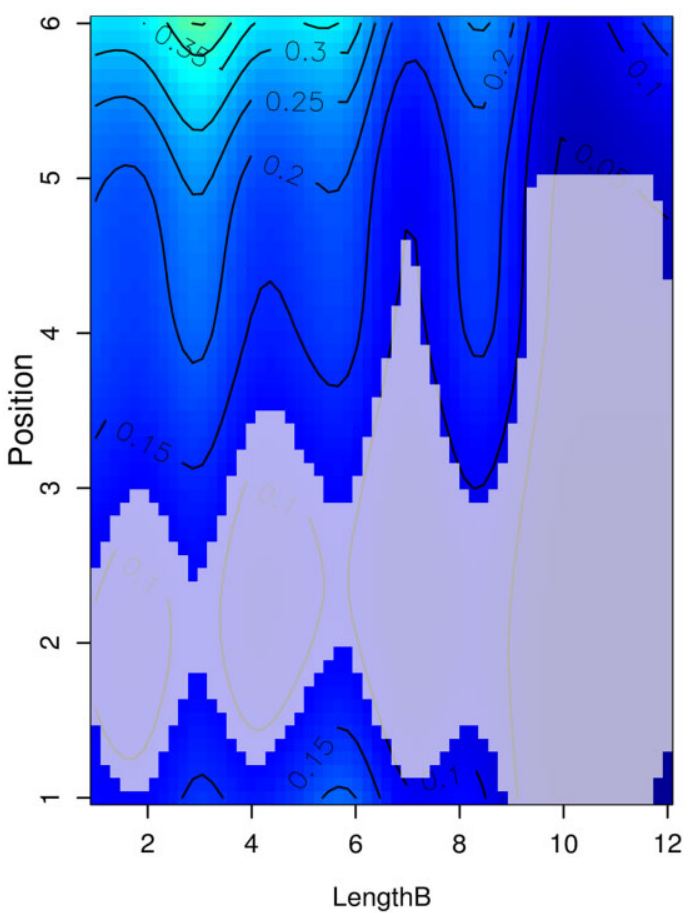

(B) Position X WMC

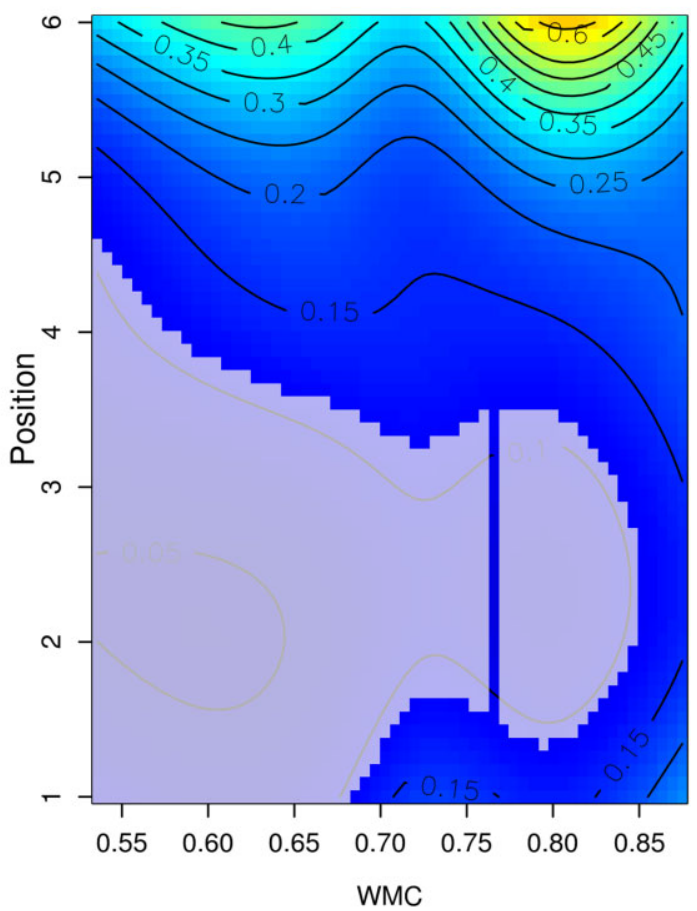

Probability of correct recall

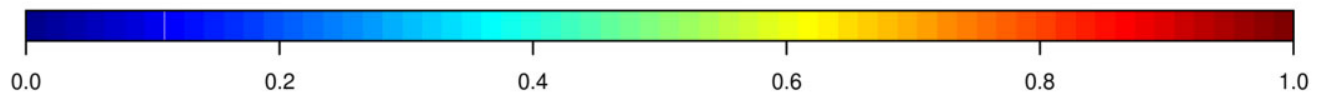

Figure 3. Results of the behavioral analysis. A: Effect of the Position $\times$ Length interaction on the probability of correctly recalling a four-word sequence. B: Effect of the Position $\times$ WMC interaction. Bluer colors reflect smaller probabilities of correctly recalling a sequence whereas redder ones indicate higher probabilities. The numbers appearing on the contour lines are recall probabilities. Regions shaded in white included a probability of correct recall of 0 in their $95 \%$ CI.

four-word sequences presented in positions 1, 2, and 3, whereas people with a WMC score above 0.7 exhibit a small primacy effect in position 1 .

\section{ERP Results}

Discretized variables and ANOVA. Although our main goal in this paper is to demonstrate the application of nonlinear analysis using continuous variables, we first show the data treated categorically. Position was treated as having six levels while length and WMC were dichotomized as high/low based on a median split. Figure 4 shows waveforms and mean N1a amplitudes for each level of each of these three variables.

No effect of length is apparent in this figure. However, there do appear to be amplitude differences for different levels of position (middle panel) - in particular, items that appeared first in the lists show a more negative N1a than subsequent positions. As such, there may be a nonlinear relationship between position and N1a amplitude. The bottom panel also suggests the possibility of an effect of WMC, where participants with a higher WMC showed a more negative N1a than those with a lower working memory capacity.

Figure 5 shows waveforms and mean amplitudes for two- and three-way combinations of levels of the independent variables (corresponding to the two- and three-way interactions in an ANOVA model). There is a possible Length $\times$ Position interaction, where long words in first position may have elicited a more negative N1a than short words in first position. There also may be a Length $\times$ WMC interaction and a WMC $\times$ Position interaction. In the former case, shorter words may have elicited a smaller N1a in participants with a low working memory capacity. In the latter case, the position effect on N1a amplitudes may have been stronger in participants with high than low working memory capacity. Finally, there is possibly a three-way interaction (bottom panel), where long words in the first position elicited a more negative N1a in participants with low compared to high working memory capacity (the leftmost black and red bars). We performed a repeated measures ANOVA to test these observations. It revealed that only the main effect of position was reliable, $F(5,96)=6.2, p<.001$.

GAMM analysis assuming linearity. The present analysis (using only the parametric component of GAMM) simply treated each variable as continuous. Results are shown in Table 2. This model suggests that there was a significant main effect of position and a significant Length $\times$ WMC interaction. These effects are depicted in Figure 6 . The position effect shown in panel A suggests that the amplitude of 

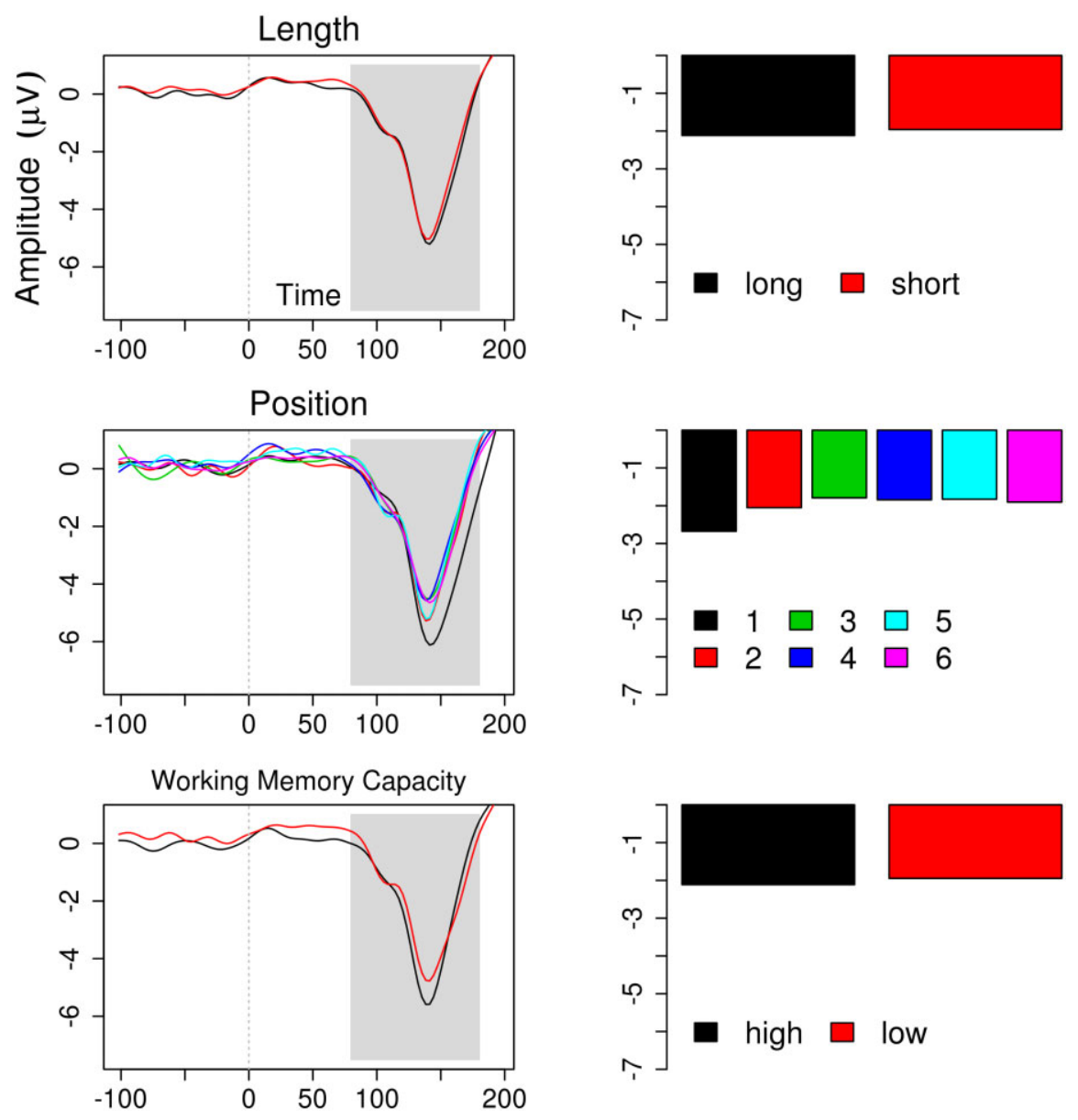

Figure 4. Waveforms (right panel) and mean amplitudes (left panel) of the N1a at electrode Fz, in the 80-180 ms window, plotted separately for discretized levels of each independent variable. The $x$ axis represents time in milliseconds and the $y$ axis is amplitude in $\mu \mathrm{V}$.

the N1a decreased (became more positive) as position increased. ${ }^{9}$ It is illustrated in panel B that the length of the second word of a sequence modulated the amplitude of the N1a. More specifically, it increased (became more negative) as the working memory capacity score of a participant increased for words 1-6 letters long, but decreased (became more positive) for words 7-12 letters long.

While it is notable that these effects were found under the assumption of linearity, Figure 4 suggested that position had a nonlinear relationship with N1a amplitude. Describing the source of this main effect would necessitate drawing inferences over the six levels of position from a relatively large number of pairwise comparisons. ${ }^{10}$ Fitting a single nonlinear function to position could simplify the description and interpretation of the effect without recourse to cumbersome post hoc testing. In addition, it may be the case that the assumption of linearity missed important structures in the data. In the next section, we explore models for which linearity is not assumed.

9. Because the N1a has a negative polarity, we refer to less negative amplitudes as "decreases" and more negative amplitudes as "increases."

10. While contrasts such as Helmert could also describe this relationship somewhat more parsimoniously, we had no a priori assumption as to the shape of the relationship between position and N1a amplitude, so the choice of appropriate contrast weights would have to be made post hoc.
Moving away from the assumption of linearity. The possibility of nonlinear relationships between our predictor variables and N1a amplitude is supported by Figure 7, in which mean N1a amplitude at each sampled level of each variable is plotted, along with a form of best-fitting nonlinear functions (LOWESS [locally weighted scatter plot smoothing] smooths). ${ }^{11}$ The length effect (top left panel) appears to be curved with relatively constant (and small) N1a amplitudes from values 1 to roughly 6 , after which N1a amplitude increases with increasing word length. The effect of position (bottom left panel) shows an opposite pattern. The amplitude of the N1a is most negative in the first position, less so in second, third, and fourth positions, and then increases slightly in the fifth and sixth positions. The WMC effect (top right panel) is more complex, with an initial increase in N1a amplitude from the lowest to low-middle WMC levels, followed by a decrease and then a subsequent increase for people with the highest WMC. We thus fitted a GAMM without assuming linearity. It included a three-way interaction Position $\times$ Length $\times \mathrm{WMC}$ and all lower

11. A LOWESS smooth uses locally weighted polynomial regression. Local is defined as a window of a certain span around each data point (e.g., 50 data points to the left and to the right). Each data point within a window is influenced (i.e., weighted) by its "neighbours." See lowess in R for more details. 

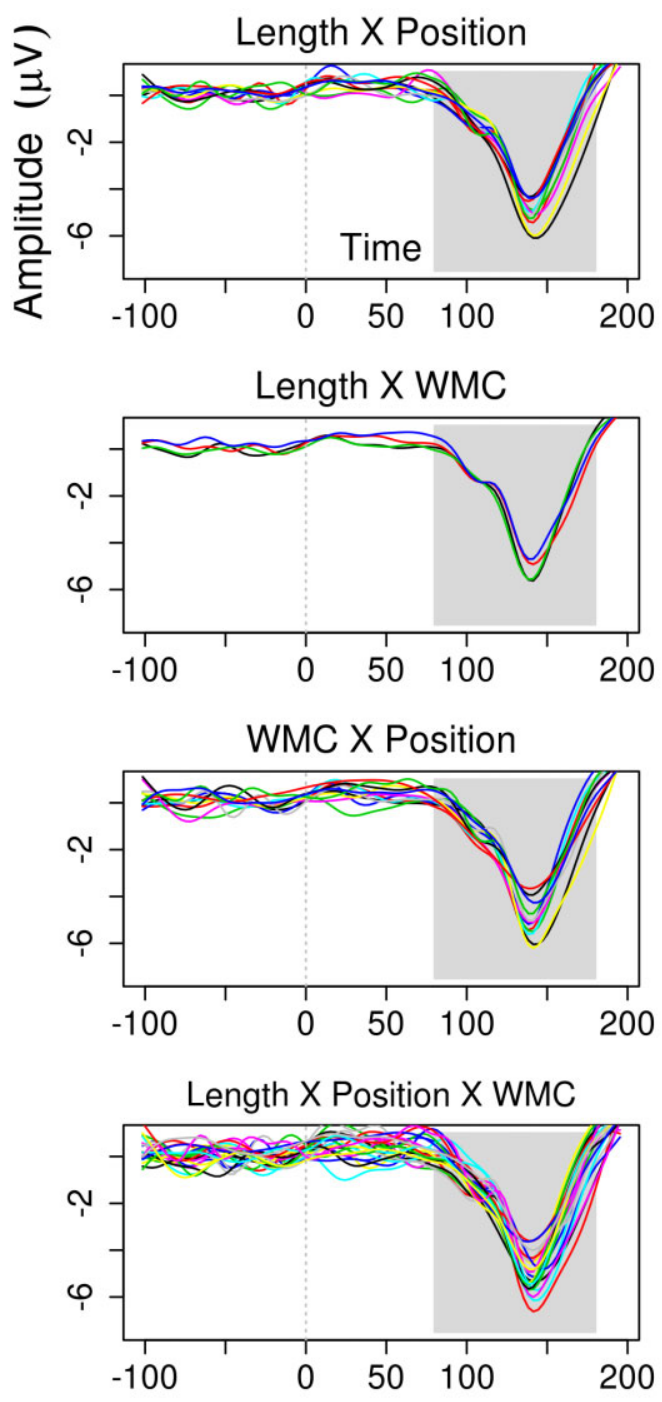
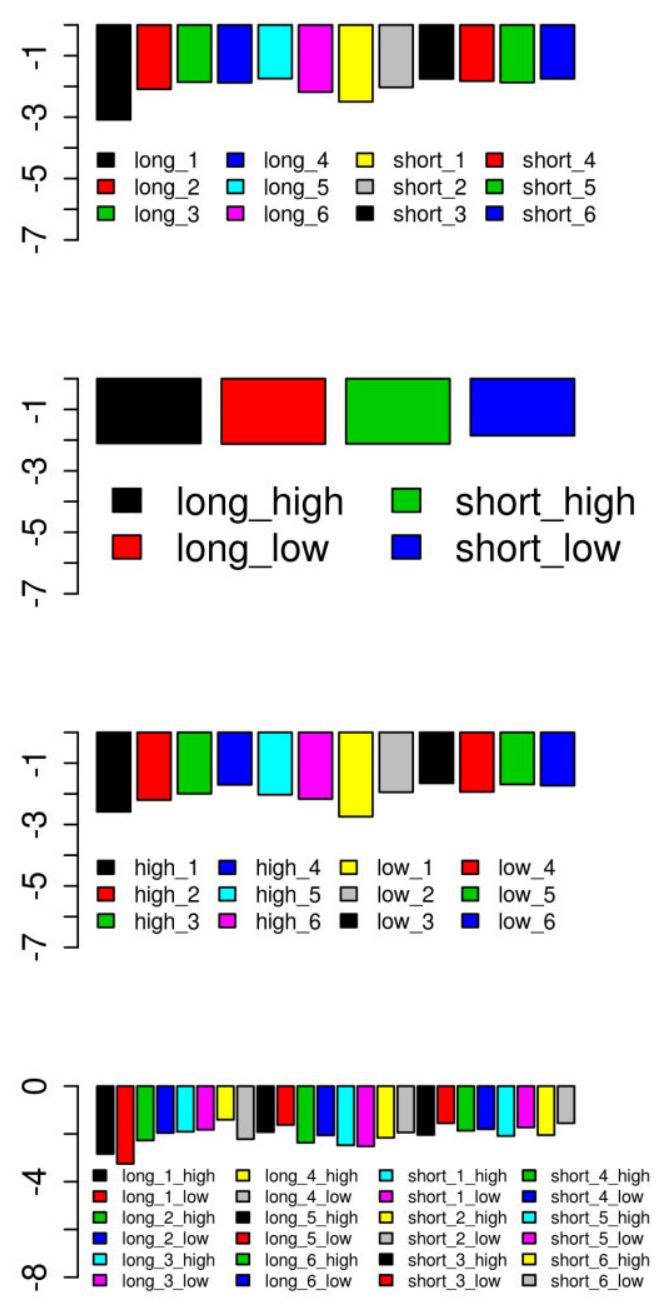

Figure 5. Two-way and three-way interactions between dichotomized levels of length, position, and WMC. Details are as in Figure 4.

order interactions and main effects, with by-subject and by-item random effects. We allowed the model to use up to 227 degrees of freedom, but GAMM deemed that 55 degrees of freedom were needed to appropriately model the data. This model was more likely given the data than the one assuming linearity (model assuming linearity: $d f=9, \mathrm{AIC}=357382$; model not assuming linearity: $d f=55$; $\mathrm{AIC}=357228$; difference: $d f=46, \mathrm{AIC}=154$ ). Results are provided in Table 3 . The plot of the three-way inter-

Table 2. Model for Which Linearity Was Assumed

\begin{tabular}{lrcrr}
\hline \hline & Estimate & $S E$ & $t$ value & $p$ value \\
\hline Pos & 0.142 & 0.018 & 8.0 & $<.001$ \\
Lngth & -0.037 & 0.029 & -1.3 & .198 \\
WMC & -0.645 & 1.376 & -0.5 & .639 \\
Pos:Lngth & 0.005 & 0.010 & 0.5 & .635 \\
Pos:WMC & -0.255 & 0.167 & -1.5 & .125 \\
Lngth:WMC & 0.472 & 0.152 & 3.1 & .002 \\
Pos:Lngth:WMC & -0.034 & 0.095 & -0.4 & .722 \\
\hline \hline
\end{tabular}

Note . Pos = position; Lngth = length; $\mathrm{WMC}=$ working memory capacity. action is shown in Figure 8. As in the analysis assuming linearity, here we found a significant main effect of position. This confirmed the pattern observed in both Figures 4 (discretized variables) and 7 (continuous variables) whereby N1a amplitudes were largest for words in the first position but were more or less consistent across subsequent positions. The lack of significant main effects for length or WMC are also consistent with the linear analysis, suggesting that, although the nonlinear curves shown in Figure 7 appeared to describe the data more closely than a straight line would have, nevertheless these relationships were not statistically reliable.

More striking differences between the linear and nonlinear analyses reside in the interaction structure of the models. While the linear model only included a significant Position $\times$ WMC interaction, the model for which linearity was not assumed contained a significant three-way interaction Position $\times$ Length $\times$ WMC. Recall that this interaction was predicted above based on our examination of the plots shown in Figure 5. The three-way interaction is depicted in Figure 8, where the Length $\times$ WMC interaction is plotted at each position. Note that regions shaded in white correspond to portions of a surface where 
(A) Position

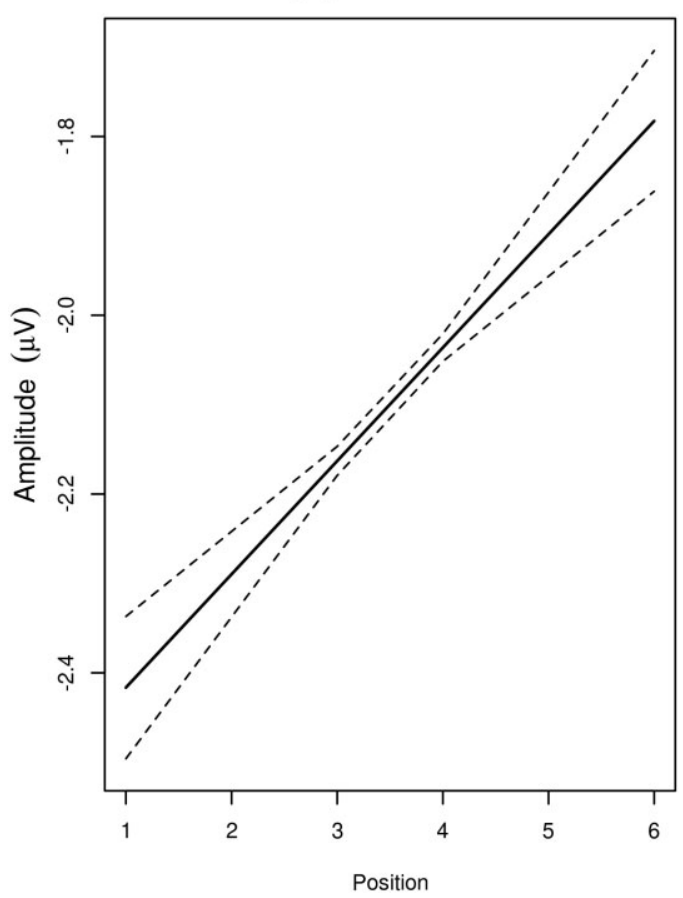

(B) Length X WMC

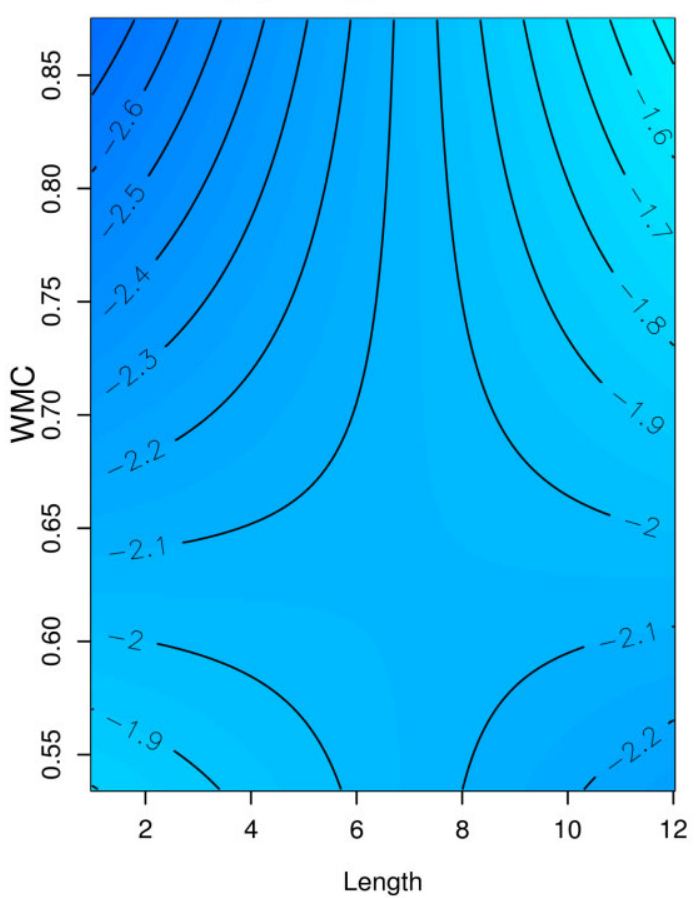

Amplitude $(\mu \mathrm{V})$

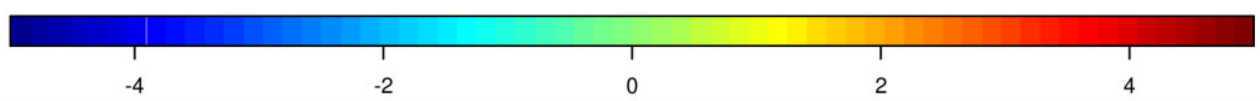

Figure 6. Results of the ERP analysis assuming linearity. A: $x$ axis is position and $y$ axis is amplitude $(\mu \mathrm{V})$. The broken lines are 95\% CIs. B: $x$ axis is length and $y$ axis is WMC. The amplitude of the N1a is shown using the same color coding as in Figure 2. The scale is provided at the bottom of the figure. The small numbers on the black lines are isovoltage lines with the voltage in microvolts provided.

the $95 \% \mathrm{CI}$ included $0 \mu \mathrm{V}$. Given the principle of marginality (Venables, 1998), we did not attempt to interpret the two-way interactions or main effects.

Figure 8 shows a complex relationship between position, length, and WMC. It is apparent that the amplitude of the N1a increased with position, and that the strongest N1a occurred at position 6 as can be evidenced by the dark blue areas in the panel for this position. Moreover, the areas shaded in white indicate that the significance of the N1a decreased as position increased; that is, the regions where the $95 \% \mathrm{CI}$ included $0 \mu \mathrm{V}$ became larger and larger. Whereas the whole surface was essentially sig-

Table 3. Model for Which Linearity Was Not Assumed

\begin{tabular}{lrrr}
\hline \hline & $e d f$ & $F$ & $p$ value \\
\hline Pos & 4 & 27.6 & $<.001$ \\
Lngth & 1 & 1.0 & .332 \\
WMC & 1 & 0.2 & .629 \\
Pos:Lngth & 9 & 2.9 & .001 \\
Pos:WMC & 12 & 4.5 & $<.001$ \\
Lngth:WMC & 4 & 2.8 & .016 \\
Pos:Lngth:WMC & 24 & 1.6 & .020 \\
\hline \hline
\end{tabular}

Note. Pos = position; Lngth = length; $\mathrm{WMC}=$ working memory capacity; $e d f=$ estimated degrees of freedom. nificant at positions 1 and 2, the N1a at positions 4, 5, and 6 was less and less reliable, especially for longer words and for participants with a WMC score below 0.65 . Starting at position 4 , the amplitude of the N1a elicited by 8- to 12-letter words was essentially $0 \mu \mathrm{V}$, while the N1a to 3-letter words became increasingly unreliable. At position 6, only 1- to 2- and 4- to 8-letter words exhibited a significant N1a, and only for participants with a WMC score above 0.65 .

In the behavioral analysis, we had found that position, length, and WMC affected the probability of a four-word sequence being correctly recalled. More specifically, (a) sequences for which the second word was longer were less likely to be correctly recalled, (b) more recently presented four-word sequences were more likely to be correctly recalled, (c) this effect was more pronounced for people with higher WMC scores, and (d) people with a lower WMC score correctly recalled four-word sequences only when they were presented in the last three positions. Our ability to relate the ERP results back to behavior, however, is seriously compromised by the fact that we conflated successfully and unsuccessfully recalled sequences. In the next section, we attempt to characterize the relationship between the probability of recall of a four-word sequence and N1a amplitudes. We additionally investigate whether the length of the second word of a sequence, the working memory capacity of a participant, and the position in which a sequence was presented modulated this relationship. 

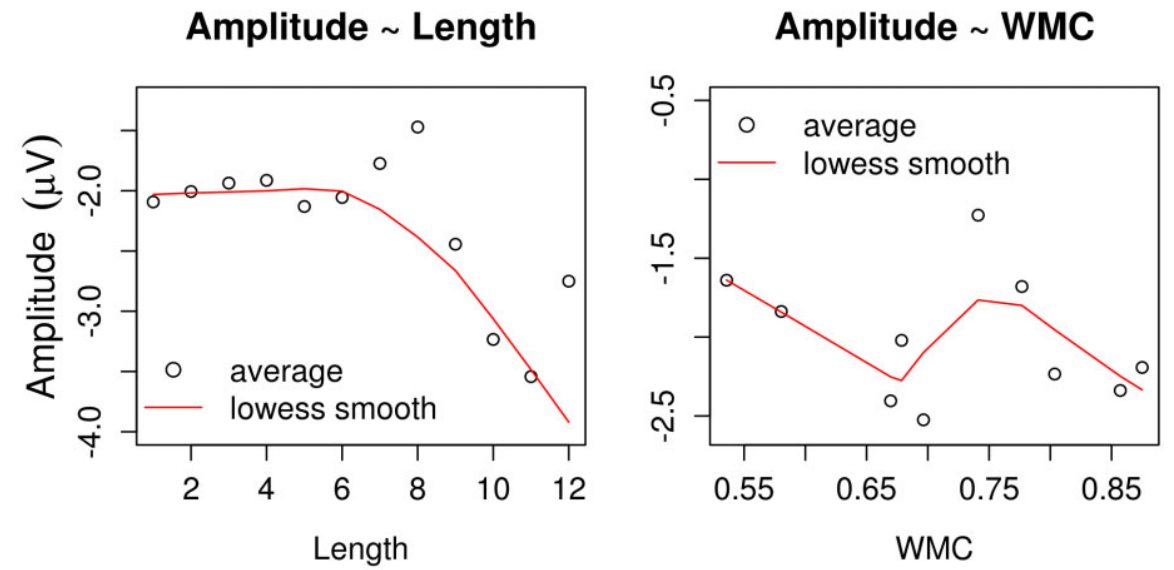

Amplitude Position

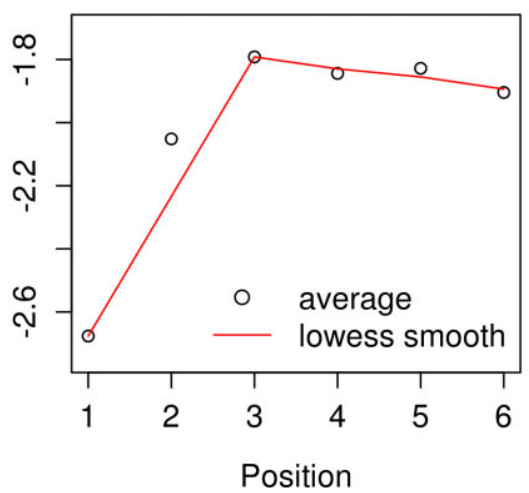

Figure 7. N1a amplitude averages as a function of each one of the predictor variables, treated as continuous (in contrast with the discretized forms of these variables shown in Figure 4). The black circles are the mean amplitude at each measured level of each variable. The black lines are LOWESS smooths of the averages (red lines in online version).

Linking behavior and scalp-recorded potentials. In order to relate the behavioral and ERP results, we first computed the probability of recall of each four-word sequence for each participant from the model obtained in the behavioral analysis. We subsequently fitted a GAMM that included a four-way interaction Position $\times$ Length $\times$ WMC $\times$ Probability of Recall to N1a amplitudes. Results are provided in Table 4 as well as in Figure 9. This model was much more likely than the previous one (model with three-way interaction: $d f=55, \mathrm{AIC}=357228$; model with four-way interaction: $d f=106, \mathrm{AIC}=351639$; difference: $d f=51, \mathrm{AIC}=5589$ ). The four-way interaction was significant, $F(40,53229)=0.4$, $p<.001$, thus suggesting that the N1a was not only affected by position, length, and WMC, but was also modulated by probability of recall. In Figure 9, each row corresponds to one of the quantiles of the probability of recall variable $(0,0.16,0.25,0.39$, and 0.92$)$ whereas each column corresponds to one of five length values $(1,4$, 6, 9, and 12). The most noteworthy feature in Figure 9 is the increase of N1a amplitudes with the probability of correctly recalling a four-word sequence. Specifically, it was equal to $0 \mu \mathrm{V}$ when the probability of recall was 0 , but increased to $-15 \mu \mathrm{V}$ at position 1 for longer words in people with higher WMC scores when the recall probability was 0.92 . It is known that the N1a is associated with attention, and that attended items typically elicit higher N1a amplitudes (e.g., Luck, 2005; Penolazzi et al., 2007, and references cited therein). If the N1a indexes the amount of attentional resources allocated to encoding a sequence, then it can be concluded that the successful recall of a four-word sequence is associated with the allocation of greater amounts of attentional resources during the encoding phase. Furthermore, longer sequences presented in the first few positions required greater amounts of attention in order to be correctly recalled, and people with higher WMC scores were able to allocate more attentional resources to these items than people with lower WMC scores.

\section{Discussion}

Relaxing the assumption of linearity enabled us to gain a better understanding of the data by capturing important structure that was overlooked in the model for which linearity was assumed. Indeed, only a main effect of Length and a Position $\times$ WMC interaction were uncovered in this model, whereas the one for which linearity was not assumed revealed a significant three-way interaction Position $\times$ Length $\times$ WMC. Moreover, even if these interactions would have reached significance in the model assuming linearity, the association strength between N1a amplitudes and these interactions (as indexed by the percentage of deviance explained, a type of effect size) would have been much lower than in the one for which linearity was not assumed, as can be seen in Table 5 .

In the model for which linearity was not assumed, the position main effect and the Length $\times$ WMC interaction accounted for 
Position 1

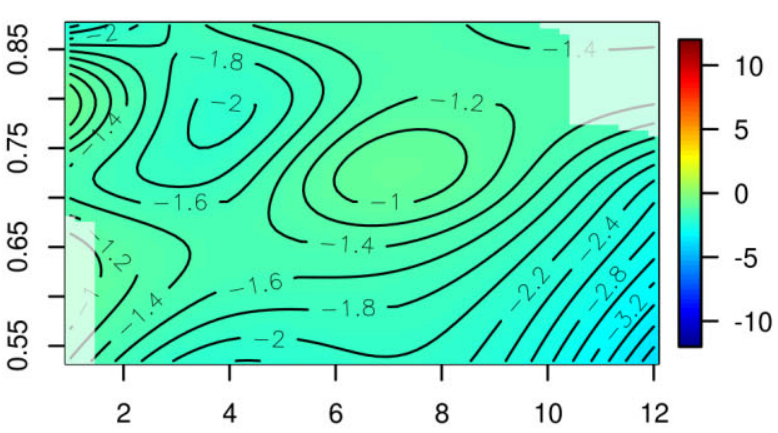

Length (\# letters)

Position 3

Amplitude ( $\mu \mathrm{V})$

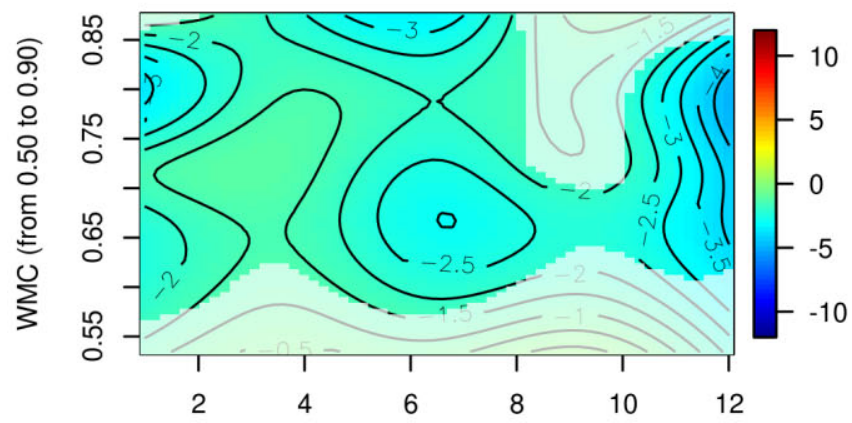

Position 5 Amplitude $(\mu \mathrm{V})$

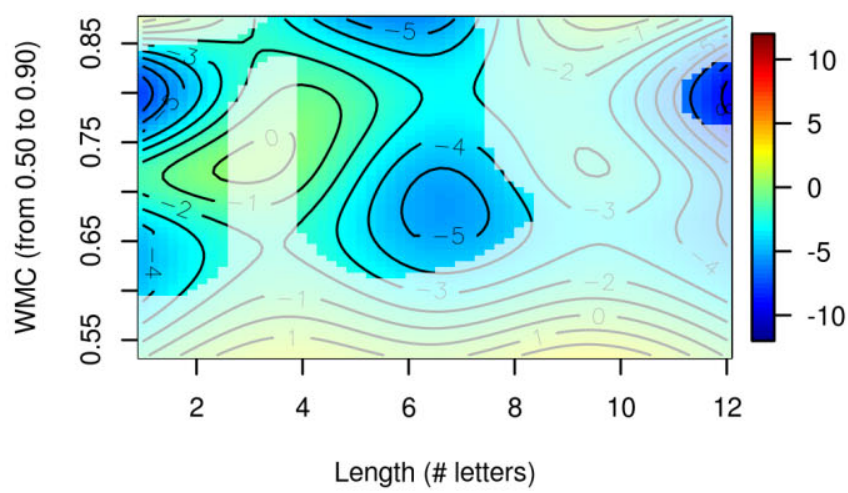

Position 2 Amplitude $(\mu \mathrm{V})$

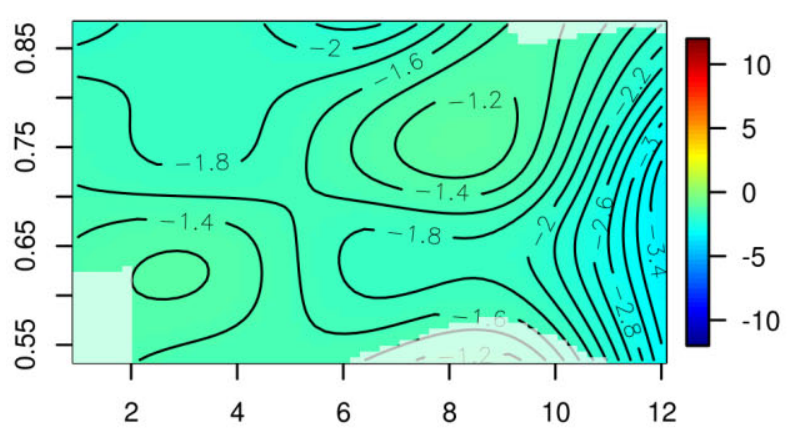

Position 4

Amplitude $(\mu \mathrm{V})$

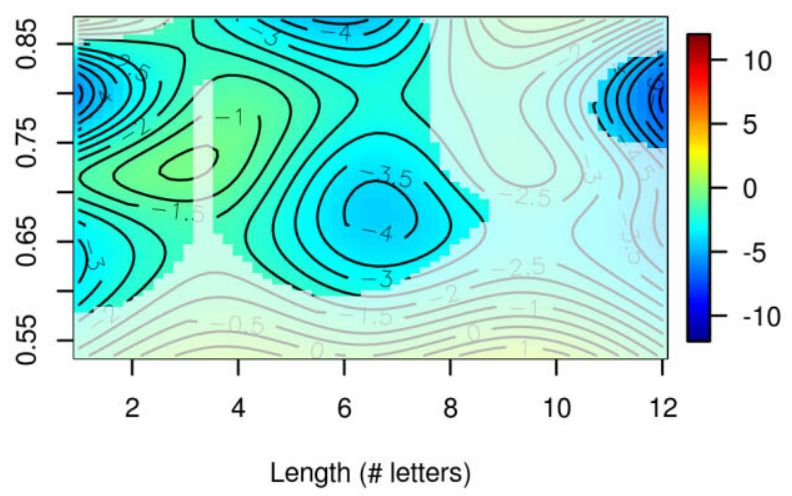

Position 6 Amplitude $(\mu \mathrm{V})$

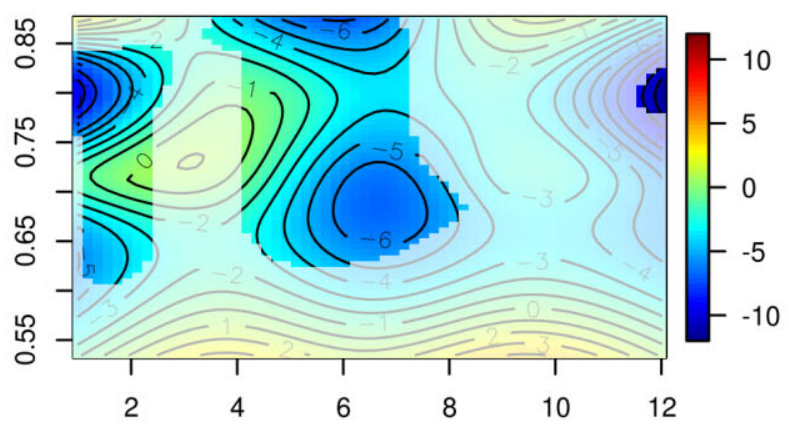

Figure 8. Results of the GAMM analysis where linearity was not assumed. Each panel shows the Length $\times$ WMC interaction at each position. The amplitude of the N1a is shown using the same color coding as in Figure 2. The scale is provided at the left of the figure. The small numbers on the black lines are isovoltage lines with the voltage in microvolts provided. Regions shaded in white correspond to portions of the smooth that included $0 \mu \mathrm{V}$ within the surface's $95 \%$ CI.

roughly twice as much deviance as in the one assuming linearity (i.e., double the effect size). Moreover, the Position $\times$ Length $\times$ WMC in the former model accounted for 337 times more deviance than the same interaction in the latter model (i.e., this effect was 337 times greater). It is important to note that the extra amount of deviance explained by the model for which linearity was not assumed is not due to data overfitting. Indeed, the effects depicted in Figures 8 and 9 were obtained by penalized regression and GCV-a data-driven approach that strikes a balance between under- and oversmoothing the data. If the effects should have been a straight line, GAMM would have penalized them to that extent, as it did in the examples provided in Figure 1B,D.

By not assuming linearity, we were also able to push our understanding of the data even further by allowing the Length $\times$ WMC smooth surfaces at each position to vary according to the probability of correct recall of a four-word sequence. The results obtained from this model-shown in Figure 9-were in close agreement with the continuous averages illustrated in Figure 7. More specifically, the N1a (a) increased as length increased, (b) was smaller for people with intermediate WMC scores (for low recall probabilities), and (c) 
Table 4. Linking Behavioral and ERP Data

\begin{tabular}{lrrr}
\hline \hline & $e d f$ & $F$ & $p$ value \\
\hline Pos & 4 & 8.2 & $<.001$ \\
Lngth & 1 & 2.7 & .100 \\
WMC & 1 & 0.2 & .693 \\
PrRec & 3 & 3.1 & .019 \\
Pos:Lngth & 5 & 3.4 & .002 \\
Pos:WMC & 11 & 2.9 & $<.001$ \\
Lngth:WMC & 1 & 2.3 & .128 \\
Pos:PrRec & 5 & 2.5 & .019 \\
Lngth:PrRec & 1 & 2.2 & .141 \\
WMC:PrRec & 1 & 1.1 & .317 \\
Pos:Lngth:WMC & 12 & 0.6 & .903 \\
Pos:Lngth:PrRec & 3 & 3.3 & .010 \\
Pos:WMC:PrRec & 12 & 1.2 & .264 \\
Lngth:WMC:PrRec & 6 & 2.2 & .031 \\
Pos:Lngth:WMC:PrRec & 40 & 0.4 & $<.001$ \\
\hline \hline
\end{tabular}

Note. Pos = position; Lngth = length; $\mathrm{WMC}=$ working memory capacity; $\operatorname{PrRec}=$ probability of correct recall; $e d f=$ estimated degrees of freedom.

was greater in the first few positions. In the remainder of the discussion, we tackle a few questions that may have arisen while reading the paper.

\section{When Can a Continuous Variable Be Discretized?}

As mentioned in the introduction, discretizing an inherently continuous variable comes at the cost of decreasing statistical power, increasing the probability of false positives, and increasing the probability of false negatives (Cohen, 1983; McCallum et al., 2002). If for some reason or another a researcher is interested in dichotomizing a variable, this should be done on the results of an analysis where continuous variables were used and linearity was not assumed. A threshold for category membership can then be derived from the model-predicted values.

\section{Hypothesis Testing and Exploratory Analyses}

It is often believed that not assuming linearity implies that one is conducting an exploratory analysis. Although it may be true in some cases, an analysis in which linearity is not assumed may just as well be based on a priori hypotheses. Both types of analyses can be performed with the same analysis pipeline used here. That is, a

Table 5. Percentage of the Deviance Explained by Each Fixed-Effect Term of the Model Assuming and Not Assuming Linearity

\begin{tabular}{lcc}
\hline \hline & $\begin{array}{c}\text { Assuming } \\
\text { linearity }\end{array}$ & $\begin{array}{c}\text { Not assuming } \\
\text { linearity }\end{array}$ \\
\hline Pos & $\mathbf{0 . 1 1 5 4 0 0}$ & $\mathbf{0 . 2 0 4 8 0 0}$ \\
Lngth & 0.000200 & 0.000000 \\
WMC & 0.000100 & 0.000200 \\
Pos:Lngth & $\mathbf{0 . 0 0 0 2 0 0}$ & $\mathbf{0 . 0 7 3 0 0 0}$ \\
Pos:WMC & $\mathbf{0 . 0 0 3 4 0 0}$ & $\mathbf{0 . 1 3 6 4 0 0}$ \\
Lngth:WMC & $\mathbf{0 . 0 1 7 6 0 0}$ & $\mathbf{0 . 0 3 5 1 0 0}$ \\
Pos:Lngth:WMC & $\mathbf{0 . 0 0 0 4 0 0}$ & $\mathbf{0 . 1 3 4 8 0 0}$ \\
\hline \hline
\end{tabular}

Note. Bold face indicates significance in the model for which linearity was not assumed. Overall, the model for which linearity was assumed accounted for $2.75 \%$ of the variance, whereas the one for which it was not assumed accounted for $2.88 \%$ of the variability in the data (including random effects). hypothesis can be deemed true or false by virtue of the effect of interest being present or not in the most likely model.

\section{Overfitting}

It is the belief of some researchers that, once the assumption of linearity is relaxed, the risk of fitting idiosyncratic features of the data is greatly enhanced. Given that GAMM uses GCV to strike a balance between over- and underfitting the data, the fitting of idiosyncratic features is unlikely to arise (see section Generalized Cross Validation in supporting information "What's Under the Hood: A Brief Introduction to GAMM" for more details and some examples). An example of this was shown in Figure 1 (i.e., GAMM did not overfit the data). The analysis pipeline also includes the viewing of a smooth's 95\% CI, which further protects against overfitting. Portions of the smooth for which 0 (the baseline) is included in the confidence interval are considered to be flat (however wiggly they may be).

\section{Sample Size}

It is sometimes thought that in order to perform mixed-effects modeling one needs a sufficient number of observations. One should not forget that a repeated measures ANOVA is also a mixed-effects model, which is often used to model small data sets without any problems. The reason we analyze our data at the single-trial level is not because we need a large sample size to perform mixed-effects modeling, but rather to account for sources of bias that would otherwise be lost during the averaging process and irreversibly affect the averages. For example, it affords us the possibility to account for unequal sample sizes between groups, a situation that commonly arises in ERP data (some trials and/or channels are usually removed). If we were to aggregate the data as is traditionally done, it would appear to contain an equal number of data points in each cell, but in fact the averages would be biased (i.e., they would have been obtained from unequal sample sizes). Another reason regarding the removal of outlier data points: If the data is unaveraged, data points with undue influence can be removed based on model residuals, thus making the results more generalizable. If the data is first averaged, outlier data points will have irreversibly affected the averages. In our experience, the need to average the data has only come about for computational tractability.

Note that an analysis for which linearity is not assumed can always be used whatever the size of the data (and still find significant effects). Some examples of this using real data sets with sample sizes between 56 and 180 are provided in file chunk25smallSampleSizeExamples.R (which can be found in the .Rnw file or in folder nlEEG/vignettes/Rcode_chunks of supporting information nlEEG).

\section{Model Convergence Problems}

Model convergence problems are most likely to arise when (a) the researcher is attempting to fit a model with too many predictor variables relative to the number of data point in the data, (b) there are too many redundant predictor variables (high collinearity), and/or (c) the random-effects structure is too complex. In the first case, one should not include in the model more predictor variables than the sample size divided by 15 (e.g., 990 data points $/ 15=66$ variables). In the second case, the researcher should deal with the collinearity present in their data in some way or another (see, e.g., Tremblay \& Tucker, 2011). In 

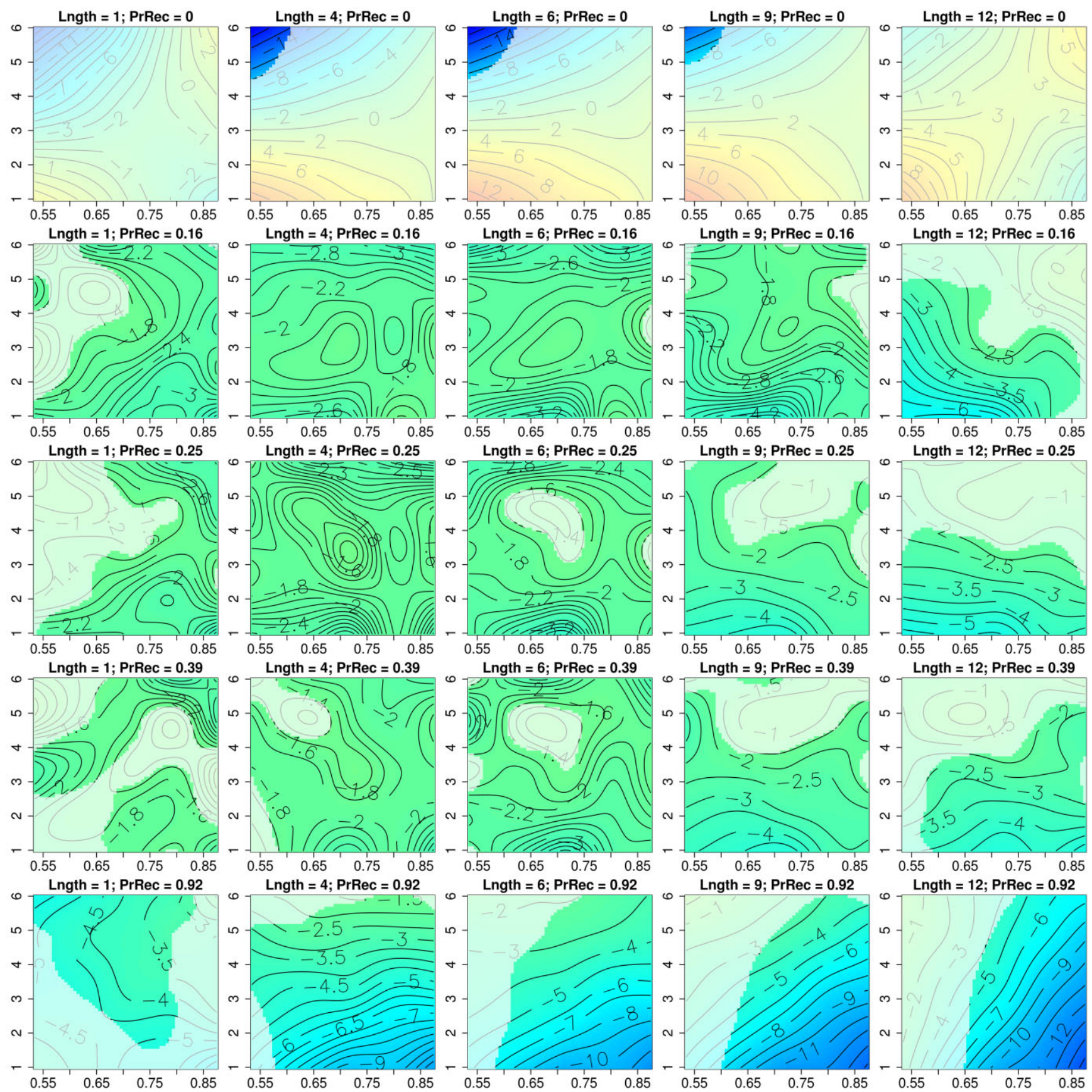

Figure 9. Four-way interaction Position $\times$ Length $\times$ WMC $\times$ Probability of Correct Recall. Each panel shows the WMC $\times$ Position model predicted N1a amplitudes for one of five lengths (columns) and probability of recall values (rows). In each panel, the $x$ and $y$ axes are WMC and position, respectively. Lngth $=$ length; PrRec $=$ probability of recall. Details are as for Figure 8.

the third case, the researcher should fit his or her model with simpler and simpler random-effects structures until the model converges. The most important random effects to include in a model are crossed by subject and by item random intercepts, which in our experience are always warranted, as well as by subject adjustments for regions of interest (to account for both individual variation in the scalp distribution of the effects as well as individual spatial correlations between levels of regions of interest; Newman, Tremblay, Nichols, Neville, \& Ullman, 2012). Note that, in our experience, models including these random effects always converge. In some analyses of ERP data, adding by-item random intercepts, and especially by-item random smooths, results in models that are computationally intractable. This forces us, unfortunately, to omit them from our models. With smaller sample sizes, computational tractability becomes less of a problem and one can usually include these random effects.

\section{Conclusion}

In order to deal with the complexity of ERP data, it is very common to assume that the relationship between ERP amplitudes/ 
latencies and independent variables is linear. This assumption does not always hold, however. Although in some ERP studies the assumption of linearity has been relaxed, these studies have typically employed the common practice of discretizing continuous variables, which has been shown elsewhere to be suboptimal.

In this paper, we have demonstrated how nonlinearities in ERP data can be modeled using generalized mixed-effects modeling in $\mathrm{R}$. This revealed that the assumption of linearity may lead one to draw incorrect inferences from his/her ERP data. More specifically, we have illustrated how the assumption of linearity may (a) increase the probability of false negatives; (b) if a relationship is obtained, provide a limited or incorrect representation of the true relationship between variables; and (c) underestimate the strength of the association between variables.
In spite of the clear potential advantage of nonlinear analyses in capturing the true structure of the data, the greatest limitation of this technique is probably the added complexity in interpreting the results. In the present case, we have demonstrated that this complexity is not necessarily uninterpretable, however, and indeed the results provide a much richer understanding of the data than could be provided by linear assumptions.

On a closing note, whether or not a researcher predicts that the relationship between a response variable and an independent variable is linear, if the independent variable is continuous and has an adequate distribution, we believe that linearity should never be assumed. If the relationship is in fact linear, GAMM will conclude that it is linear (as was illustrated in Figure 1D), and results will be that much more convincing. If the relationship emerges as nonlinear, then theory can be adjusted.

\section{References}

Akaike, H. (1973). Information theory and an extension of the maximum likelihood principle. In B. Petrov \& F. Csaki (Eds.), 2nd International Symposium on Information Theory (pp. 267-281). Budapest, Hungary.

Assadollahi, R., \& Pulvermüller, F. (2003). Early influences of word length and frequency: A group study using MEG. NeuroReport, 14, 11831187. doi: 10.1097/01.wnr.0000075305.76650.60

Baayen, R. H. (2008). Analyzing linguistic data: A practical introduction to statistics using R. Cambridge, UK: Cambridge University Press. doi: 10.1017/S0305000909990080

Baayen, R. H., Davidson, D. J., \& Bates, D. M. (2008). Mixed-effects modeling with crossed random effects for subjects and items. Journal of Memory and Language, 59, 390-412. doi: 10.1016/j.jml.2007 .12 .005

Baayen, R. H., Kuperman, V., \& Bertram, R. (2010). Frequency effects in compound processing. In S. Scalise \& I. Vogel (Eds.), Compounding. (pp. 257-270). Amsterdam, The Netherlands: John Benjamins.

Bagiella, E., Sloan, R., \& Heitjan, D. (2000). Mixed-effects models in psychophysiology. Psychophysiology, 37, 13-20. doi: 10.1111/14698986.3710013

Boutheina, J., Coutya, J., Langer, C., \& Roy, S. (2009). From upright to upside-down presentation: A spatio-temporal ERP study of the parametric effect of rotation on face and house processing. BMC Neuroscience, 10, 1-17. doi: 10.1186/1471-2202-10-100

Burnham, K., Anderson, D., \& Huyvaert, K. (2011). AIC model selection and multimodel inference in behavioral ecology: Some background, observations, and comparisons. Behavioral Ecology and Sociobiology, 65, 25-35. doi: 10.1007/s00265-010-1029-6

Carbon, C.-C., Schweinberger, S. R., Kaufmann, J. M., \& Leder, H. (2005). The Thatcher illusion seen by the brain: An event-related brain potentials study. Cognitive Brain Research, 24, 544-555. doi: 10.1016/ j.cogbrainres.2005.03.008

Cohen, J. (1983). The cost of dichotomization. Applied Psychological Measurement, 7, 249-254. doi: 10.1177/014662168300700301

Daneman, M., \& Carpenter, P. A. (1980). Individual differences in working memory and reading. Journal of Verbal Learning and Verbal Behavior, 19, 450-466. doi: 10.1037/0278-7393.9.4.561

Davies, M. (2004). BYU-BNC: The British national corpus. Available online at http://corpus.byu.edu/bnc

Faraway, J. J. (2005). Extending the linear model with R: Generalized linear, mixed effects and nonparametric regression models. New York, NY: Chapman \& Hall. doi: 10.1007/s00180-009-0152-1

Fletcher, W. H. (2008). Phrases in English. Available online at http:// pie.usna.edu/index.html

Gelman, A., \& Hill, J. (2007). Data analysis using regression and multilevel/hierarchical models. Cambridge, UK: Cambridge University Press. doi: 10.1017/CBO9780511790942

Gratton, G., Coles, M., \& Donchin, E. (1983). A new method for off-line removal of ocular artifact. Electroencephalography and Clinical Neurophysiology, 55, 468-484. doi: 10.1016/0013-4694(83)90135-9

$\mathrm{Gu}, \mathrm{C}$. (2002). Smooth splines ANOVA models. New York, NY: Springer.
Gu, C., \& Wahba, G. (1991). Minimizing GCV/GML scores with multiple smoothing parameters via the Newton method. SIAM Journal of Scientific and Statistical Computing, 12, 383-398.

Hastie, T., \& Tibshirani, R. (1990). Generalized additive model regression. New York, NY: Chapman \& Hall.

Hauk, O., Davis, M., Ford, M., Pulvermüller, F., \& Marslen-Wilson, W. (2006). The time course of visual word recognition as revealed by linear regression analysis of ERP data. NeuroImage, 30, 1383-1400. doi: 10.1016/j.clinph.2003.12.020

Hauk, O., \& Pulvermüller, F. (2004). Effects of word length and frequency on the human event-related potential. Clinical Neurophysiology, 115, 1090-1103. doi: 10.1016/j.clinph.2003.12.020

Hofman, D. A., \& Gavin, M. B. (1998). Centering decisions in hierarchical linear models: Implications for research in organizations. Journal of Management, 24, 623-641. doi: 10.1177/014920639802400504

Inui, K., Urakawa, T., Yamashiro, K., Otsuru, N., Nishihara, M., Takeshima, Y., ... Kakigi, R. (2010). Non-linear laws of echoic memory and auditory change detection in humans. BMC Neuroscience, 11, 1-13. doi: 10.1186/1471-2202-11-80

Jones, T., \& Oberauer, K. (2013). Serial-position effects for items and relations in short-term memory. Memory, 21, 347-365. doi: 10.1080/ 09658211.2012 .726629

Keele, L. (2008). Semiparametric regression for the social sciences. New York, NY: Chapman \& Hall/CRC. doi: 10.1002/9780470998137

Kreft, I., \& De Leeuw, J. (1998). Introducing multilevel modeling. London, UK: Sage. doi: 10.4135/9781849209366

Kriegeskorte, N., Simmons, W., Bellgowan, P., \& Baker, C. (2009). Circular analysis in systems neuroscience: The dangers of double dipping. Nature Neuroscience, 12, 535-540. doi: 10.1038/nn.2303

Kryuchkova, T., Tucker, B. V., Wurm, L. H., \& Baayen, R. H. (2012). Danger and usefulness are detected early in auditory lexical processing: Evidence from electroencephalography. Brain and Language, 122, 81-91. doi: 10.1016/j.band1.2012.05.005

Lin, X., \& Zhang, D. (1999). Inference in generalized additive mixed models by using smoothing splines. Journal of the Royal Statistical Society, 61, 381-400. doi: 1369-7412/99/61381

Luck, S. J. (2005). An introduction to the event-related potential technique. Cambridge, MA: MIT Press.

Marra, G., \& Wood, S. N. (2012). On $p$-values for smooth components of an extended generalized additive model. Scandinavian Journal of Statistics, 39, 53-74. doi: 10.1111/j.1467-9469.2011.00760.x

McCallum, R. C., Zhang, S., Preacher, K. J., \& Rucker, D. D. (2002). On the practice of dichotomization of quantitative variables. Psychological Methods, 7, 19-40. doi: 10.1037/1082-989X.7.1.19

Murphy, K., Roodenrys, S., \& Fox, A. (2006). Event-related potential correlates of the word length effect in working memory. Brain Research, 1112, 179-190. doi: 10.1016/j.brainres.2006.07.007

Newman, A., Tremblay, A., Nichols, E. S., Neville, H. J., \& Ullman, M. (2012). The influence of language proficiency on lexical semantic processing in native and late learners of English. Journal of Cognitive Neuroscience, 25, 1205-1223. doi: 10.1162/jocn_a_00143 
Oldfield, R. C. (1971). The assessment and analysis of handedness: The Edinburgh inventory. Neuropsychologica, 9, 97-113. doi: 10.1016/ 0028-3932(71)90067-4

Pablos Martin, X., Deltenre, P., Rossion, B., Hoonhorst, I., \& Colin, C. (2007). Perceptual biases for rhythm: The mismatch negativity latency indexes the privileged status of binary vs non-binary interval ratios. Clinical Neurophysiology, 118, 2709-2715. doi: 10.1016/ j.clinph.2007.08.019

Penolazzi, B., Hauk, O., \& Pulvermüller, F. (2007). Early semantic context integration and lexical access as revealed by event-related brain potentials. Biological Psychology, 72, 373-388. doi: 10.1016/j .biopsycho.2006.09.008

Pinheiro, J. C., \& Bates, D. M. (2000). Mixed-effects models in S and S-plus. New York, NY: Springer.

Quené, H., \& van den Bergh, H. (2008). Examples of mixed-effects modeling with crossed random effects and with binomial data. Journal of Memory and Language, 59, 413-425. doi: 10.1016/j.jml.2008.02.002

R Development Core Team. (2013). R: A language and environment for statistical computing [Computer software manual]. Vienna, Austria. Retrieved from http://www.R-project.org

Ruppert, D., Wand, M., \& Carroll, R. (2003). Semiparametric regression. Cambridge, UK: Cambridge University Press. doi: 10.1017/ CBO9780511755453

Sahai, H., \& Ageel, M. I. (1997). Analysis of variance: Fixed, random and mixed models. Boston, MA: Birkhauser.

Sereno, S., Rayner, K., \& Posner, M. (1998). Establishing a time-line of word recognition: Evidence from eye movements and event-related potentials. NeuroReport, 9, 2195-2200. doi: 10.1097/00001756199807130-00009

Symonds, M., \& Moussalli, A. (2011). A brief guide to model selection, multimodel inference and model averaging in behavioural ecology using Akaike's information criterion. Behavioral Ecology and Sociobiology, 65, 13-21. doi: 10.1007/s00265-010-1037-6

Tremblay, A. (2009). Processing advantages of lexical bundles: Evidence from self-paced reading, word and sentence recall, and free recall with event-related brain potential recordings. (Doctoral dissertation). University of Alberta, Edmonton, $\mathrm{AB}$, Canada.

Tremblay, A., \& Baayen, R. H. (2010). Holistic processing of regular four-word sequences: A behavioral and ERP study of the effects of structure, frequency, and probability on immediate free recall. In D. Wood (Ed.), Perspectives on formulaic language: Acquisition and communication (pp. 151-173). London, UK: Continuum.

Tremblay, A., \& Tucker, B. V. (2011). The effects of n-gram probabilistic measures on the recognition and production of four-word sequences. Mental Lexicon, 6, 302-324. doi: 10.1075/ml.6.2.04tre
Venables, W. (1998). Exegeses on linear models. Paper presented at the S-PLUS User's Conference, Washington, DC. Retrieved from http:// www.stats.ox.ac.uk/pub/MASS3/Exegeses.pdf

Vossen, H., van Breukelen, G., Hermens, H., van Os, J., \& Lousberg, R. (2011). More potential in statistical analyses of event-related potentials: A mixed regression approach. International Journal of Methods in Psychiatric Research, 20, e56-e68. doi: 10.1002/mpr.348

Webb, S. J., Long, J. D., \& Nelson, C. A. (2005). A longitudinal investigation of visual event-related potentials in the first year of life. Developmental Science, 8, 605-616. doi: 10.1111/j.1467-7687 .2005.00452.x

Wood, S. N. (2006). Generalized additive models. New York, NY: Chapman \& Hall/CRC.

Wood, S. N. (2012). mgcv: Mixed GAM computation vehicle with GCV/ AIC/REML smoothness estimation ( $\mathrm{R}$ package version 1.7-22) [Computer software manual]. Retrieved from http://CRAN.R-project.org/ package $=$ mgcv.

Wood, S. N. (2013). On $p$-values for smooth components of an extended generalized additive model. Biometrika, 100, 221-228. doi: 10.1093/ biomet/ass048

Wu, H., \& Zhang, J. (2006). Nonparametric regression methods for longitudinal data analysis. Hoboken, NJ: Wiley. doi: 10.1002/ 0470009675

Wu, L. (2010). Mixed effects models for complex data. New York, NY: Chapman \& Hall.

Zuur, A. F., Ieno, E. N., Walker, N. J., Saveliev, A. A., \& Smith, G. M. (2009). Mixed effects models and extensions in ecology with R. New York, NY: Springer.

(Received November 14, 2013; ACCEPTED June 23, 2014)

\section{Supporting Information}

Additional supporting information may be found in the online version of this article:

Appendix S1: nlEEG

Appendix S2: "What's Under the Hood: A Brief Introduction to GAMM" 